\title{
Automated Concolic Testing of Smartphone Apps
}

\author{
Saswat Anand \\ Georgia Institute of \\ Technology \\ saswat@gatech.edu
}

\author{
Mayur Naik \\ Georgia Institute of \\ Technology \\ naik@cc.gatech.edu \\ Mary Jean Harrold \\ Georgia Institute of \\ Technology \\ harrold@cc.gatech.edu
}

\author{
Hongseok Yang \\ University of Oxford \\ hongseok.yang@cs.ox.ac.uk
}

\begin{abstract}
We present an algorithm and a system for generating input events to exercise smartphone apps. Our approach is based on concolic testing and generates sequences of events automatically and systematically. It alleviates the pathexplosion problem by checking a condition on program executions that identifies subsumption between different event sequences. We also describe our implementation of the approach for Android, the most popular smartphone app platform, and the results of an evaluation that demonstrates its effectiveness on five Android apps.
\end{abstract}

\section{Categories and Subject Descriptors}

D.2.5 [Software Engineering]: Testing and DebuggingSymbolic execution, Testing tools

\section{Keywords}

Testing graphical user interfaces, Testing Event-driven programs, Smartphone apps, Android

\section{INTRODUCTION}

Mobile devices with advanced computing ability and connectivity, such as smartphones and tablets, are becoming increasingly prevalent. At the same time there has been a surge in the development and adoption of specialized programs, called apps, that run on such devices. Apps pervade virtually all activities ranging from leisure to missioncritical. Thus, there is a growing need for software-quality tools in all stages of an app's life-cycle, including development, testing, auditing, and deployment.

Apps have many features that make static analysis challenging: a vast software development kit (SDK), asynchrony, inter-process communication, databases, and graphical user interfaces (GUIs). As a result, many existing approaches for analyzing apps are based on dynamic analysis (e.g., 6, 8, 9]).

Permission to make digital or hard copies of all or part of this work for personal or classroom use is granted without fee provided that copies are not made or distributed for profit or commercial advantage and that copies bear this notice and the full citation on the first page. To copy otherwise, to republish, to post on servers or to redistribute to lists, requires prior specific permission and/or a fee.

SIGSOFT'12/FSE-20, November 11-16, 2012, Cary, North Carolina, USA. Copyright 2012 ACM 978-1-4503-1614-9/12/11 ...\$15.00.
A question central to the effectiveness of any dynamic analysis is how to obtain relevant program inputs. The most indivisible and routine kind of inputs to an app are events. A tap on the device's touch screen, a key press on the device's keyboard, and an SMS message are all instances of events. This paper presents an algorithm and a system for generating input events to exercise apps. Apps can-and in practice often do-possess inputs besides events, such as files on disk and secure web content. Our work is orthogonal and complementary to approaches that provide such inputs.

Apps are instances of a class of programs we call eventdriven programs: programs embodying computation that is architected to react to a possibly unbounded sequence of events. Event-driven programs are ubiquitous and, besides apps, include stream-processing programs, web servers, GUIs, and embedded systems. Formally, we address the following problem in the setting of event-driven programs in general, and apps in particular.

Branch-coverage Problem: Given a constant bound $k \geq 1$, efficiently compute a set of event sequences that executes each branch of an eventdriven program that can executed by some event sequence of length up to $k$.

The above problem poses two separate challenges: (1) how to generate single events and (2) how to extend them to sequences of events. We next look at each of these in turn.

Generating Single Events. Existing approaches for generating all events of a particular kind use either capturereplay techniques to automatically infer a model of the app's GUI [21, 30] or model-based techniques that require users to provide the model 33, 35. These approaches have limitations. Capture-replay approaches are tailored to a particular platform's event-dispatching mechanism but many apps use a combination of the platform's logic and their own custom logic for event dispatching. For example, where the platform sees a single physical widget, an app might interpret events dispatched to different logical parts of that widget differently. In contrast, model-based approaches are generalpurpose, but they can require considerable manual effort.

In this paper, we present a new approach, which is both general and automatic, to address this problem. The approach builds on a systematic test-input generation technique, concolic testing (also known as dynamic symbolic execution), which has made significant strides in recent years [5, 14, 29. Our approach symbolically tracks events from 
the point where they originate to the point where they are ultimately handled. The approach is thus oblivious to where and how events are dispatched.

Generating Event Sequences. Our concolic-testing approach for generating single events can be extended naturally to iteratively compute sets of increasingly longer sequences of events. An algorithm, hereafter called AlLSEQS, can generate all event sequences of length up to $k$ such that each event sequence executes a unique program path. However, AllSeqs does not scale as the value of $k$ is increased because typical programs have a large number of program paths. For instance, for a simple music player app, AlLSEQS generates 11 one-event sequences, 128 two-event sequences, 1,590 three-event sequences, and $21 \mathrm{~K}$ four-event sequences. This problem is known as the path-explosion problem [1], and, in practice, AllSeQs may fail to solve the aforementioned branch-coverage problem within a time budget for a high value of $k$.

Although several techniques have been developed in recent years to address the path-explosion problem (e.g., 1], 3, 1113, 16, 18, 19, 28]), the problem is still intractable for realworld software. In this paper, we present a new technique, ACTEvE (stands for Automated Concolic Testing of Eventdriven programs), to alleviate the path-explosion problem. ACTEVE is tailored to event-driven programs such as smartphone apps. The key insight underlying ACTEvE is a notion of subsumption relation between two event sequences. If an event sequence $\pi$ is subsumed by another event sequence $\pi^{\prime}$, then ACTEVE avoids generating sequences that are extensions of $\pi$, and instead generates extensions of $\pi^{\prime}$ only. Such pruning of $\pi$ results in compounded savings as longer event sequences are generated. We show that ACTEvE is relatively complete with respect to ALLSEQS. Specifically, ACTEve covers the same set of branches as ALLSEQS for a given upper bound on the length of event sequences ${ }^{1}$

To compute subsumption relation between event sequences, ACTEVE checks for simple data- and control-flow facts of each program execution in isolation. Compared to existing techniques for the path-explosion problem, ACTEvE has at least four distinctive characteristics that greatly improves its applicability to real-world software such as the Android system. First, it does not impose any unrealistic assumption (e.g., methods are free of side effects) about the program. Second, it does not store and match program traces or program states, which can dramatically increase the memory overhead. Third, it does not require powerful (e.g., support for quantifiers) or specialized constraint solvers to reason about path constraints of multiple program paths simultaneously. Fourth, it does not require static analysis of real-world code that often contain parts that are beyond the scope of static analysis. Despite its above-mentioned simplicity, in our empirical study, ACTEvE produced significant savings because subsumption relation between event sequences exists frequently in real apps. For example, for the previouslymentioned music player app, using $k=4$, our algorithm explores only $16 \%$ of the inputs $(3,445$ out of 21,117$)$ that ALLSEQS explores.

We have implemented our algorithm in a system for Android, the dominant smartphone app platform. Our system instruments and exercises the given app in a mobile device

\footnotetext{
${ }^{1}$ ACTEve can also be used for bounded verification of safety properties, by converting a given assert(e) statement to if (e) assert (false) and checking reachability of the assert (false) statement.
}

emulator that runs an instrumented Android SDK. This enables our system to be portable across mobile devices, leverage Android's extensive tools (e.g., to automatically run the app on generated inputs), and exploit stock hardware; in particular, our system uses any available parallelism, and can run multiple emulators on a machine or a cluster of machines. Our system also builds upon recent advances in concolic testing such as function summarization 11, generational search [15], and Satisfiability Modulo Theories (SMT) solving [7. We demonstrate the effectiveness of our system on five Android apps.

The primary contributions of this work are as follows.

1. A novel approach to systematically generate events to exercise apps. Our approach, based on concolic testing, is fully automatic and general, in contrast to existing capture-replay-based or model-based approaches.

2. An efficient algorithm, ACTEve, to solve the branchcoverage problem. Our key insight is a subsumption condition between event sequences. Checking the condition enables our ACTEvE to prune redundant event sequences, and thereby alleviate path explosion, while being complete with respect to AlLSEqS.

3. An implementation and evaluation of ACTEve in a system for Android, the dominant smartphone app platform. Our system is portable, exploits available parallelism, and leverages recent advances in concolic testing. We demonstrate the effectiveness of our system on five Android apps.

\section{OVERVIEW OF OUR APPROACH}

In this section, we illustrate our approach using an example music player app from the Android distribution. We first describe the app by discussing its source code shown in Figure 1 (Section 2.1). We then describe how we generate events to exercise this app (Section 2.2) and how we extend them to sequences of events (Section 2.3 .

\subsection{The Music Player App}

Android apps are incomplete programs: they implement parts of the Android SDK's API and lack a main method. When the music player app is started, the SDK creates an instance of the app's main activity MainActivity, and calls its onCreate () method. This method displays the main screen, depicted in Figure 2(a), which contains six buttons: rewind, play, pause, skip, stop, and eject. The method also sets the main activity as the handler of clicks to each of these buttons. The app waits for events once onCreate() finishes.

When any of the six buttons is clicked, the SDK calls the main activity's onClick() method, because the main activity was set to handle these clicks. If the eject button is clicked, this method displays a dialog, depicted in Figure 2(b), that prompts for a music file URL. If any of the other buttons is clicked, the onClick() method starts a service MusicService with an argument that identifies the button. The service processes clicks to each of the five buttons. For brevity, we show only how clicks to the rewind button are processed, in the processRewind() method. The service maintains the current state of the music player in mState. On start-up, the service searches for music files stored in the device, and hence, the state is initialized to Retrieving. Clicks to each button have effect only in certain states; for instance, clicking the rewind button has no effect unless the 


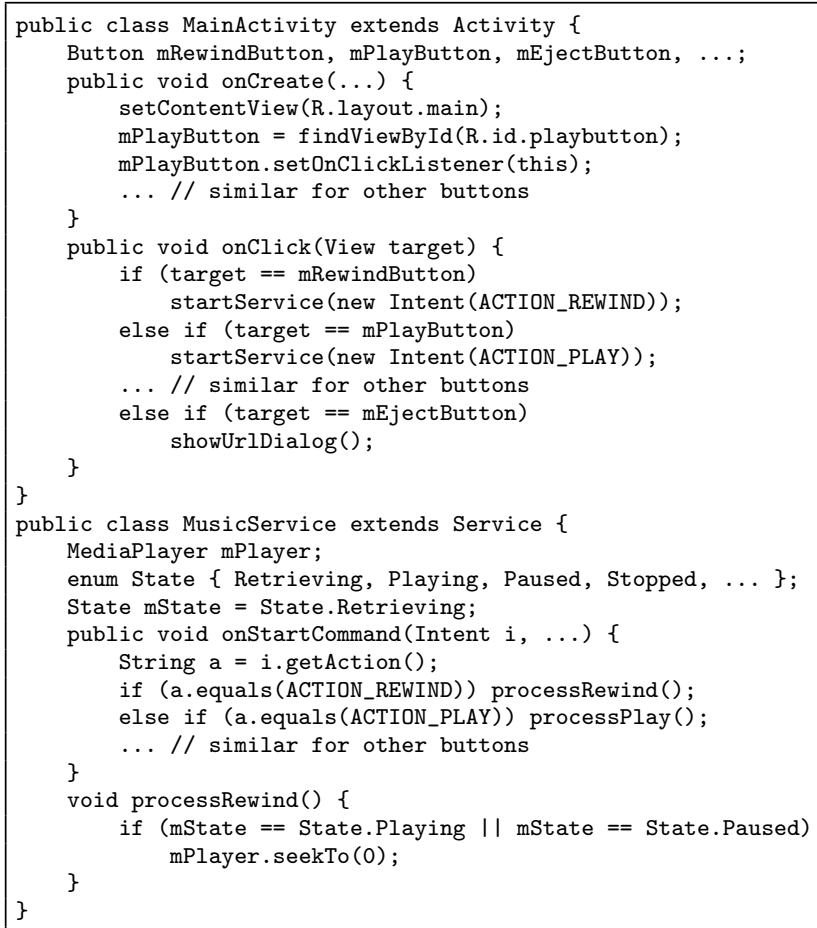

Figure 1: Source code snippet of music player app.

state is Playing or Paused, in which case processRewind() rewinds the player to the start of the current music file.

\subsection{Generating Single Events}

Our first goal is to systematically generate single input events to a given app in a given state. For concreteness, we focus on tap events, which are taps on the device's touch screen, but our observations also hold for other kinds of events, such as key presses on the device's keyboard and incoming phone calls.

Our goal is to generate tap events such that each widget on the displayed screen of the app is clicked once. In Android, the widgets on any screen of an app are organized in a tree called the view hierarchy, where each node denotes the rectangular bounding box of a different widget, and the node's parent denotes its containing widget. Figure 3 shows the view hierarchy for the main screen of the music player app depicted in Figure 2(a). Given this hierarchy, we can achieve our goal of clicking each widget once, by generating Cartesian coordinates inside each rectangle in the hierarchy and outside its sub-rectangles.

As we discussed in the Introduction, existing approaches either infer the hierarchy automatically (capture-replay [21, 30]) or require users to provide it (model-based approaches 33, 35); both have limitations. We present a new approach that is general and fully automatic. Our approach is based on concolic testing, and symbolically tracks events from the point where they originate to the point where they are handled. For this purpose, our approach instruments the Android SDK and the app under test. In the case of tap events, whenever a concrete tap event is input, this instrumentation creates a fresh symbolic tap event and propagates it alongside the concrete event. As the concrete event flows through the SDK and the app, the instrumentation tracks a constraint on the corresponding symbolic event which effec-

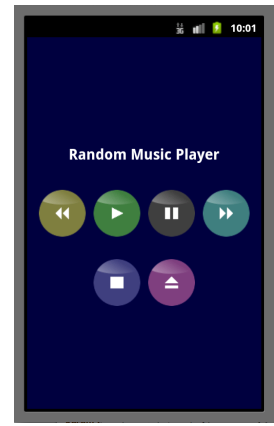

(a) Main screen.

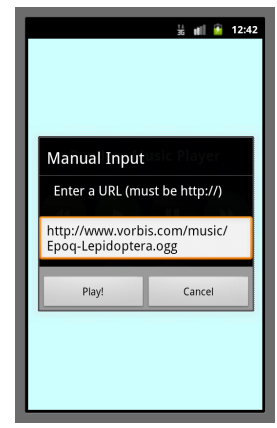

(b) Eject dialog.
Figure 2: Screen shots of music player app.

tively identifies all concrete events that are handled in the same manner. This not only lets our approach avoid generating spurious events but also enables it to exhaustively generate orthogonal events. For the main screen of our music player app, for instance, our approach generates exactly 11 tap events, one in each of the rectangles (and outside sub-rectangles) in the screen's view hierarchy depicted in Figure 3 Section 3 describes how our approach generates these events in further detail.

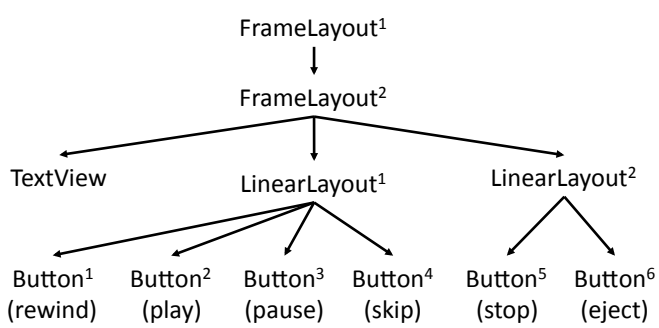

Figure 3: View hierarchy of main screen.

\subsection{Generating Event Sequences}

Our concolic testing approach for generating single events can be extended naturally to iteratively compute sets of increasingly longer sequences of events. However, as we discussed in the Introduction, the ALLSEQS concolic testing approach [14, 29] causes the computed sets to grow rapidlyfor the above music player app, AllSEQS produces 11 oneevent sequences, 128 two-event sequences, 1,590 three-event sequences, and $21 \mathrm{~K}$ four-event sequences.

We studied the event sequences produced by ALLSEQS for several Android apps and observed a significant source of redundancy. empirically that a significant percentage of events do not have any effect on the program state. We call such events read-only because we identify them by checking that no memory location is written when they are dispatched and handled. Upon closer inspection, we found diverse reasons for the prevalence of read-only events, which we describe below.

First, many widgets on any given screen of any app never react to any clicks. As shown in the view hierarchy of the main screen of the music player app (Figure 3), these include boilerplate widgets like FrameLayout and LinearLayout, which merely serve to layout other actionable widgets, such as Button, and informational widgets, such as TextView, that display non-editable texts. Thus, only 6 of 11 widgets on the main screen of the music player app 
(namely, the six buttons) are actionable, and clicks to the remaining 5 widgets constitute read-only events.

Second, many widgets that are actionable might be disabled in certain states of the app. This situation often occurs when apps wish to guide users to provide events in a certain order or when they wish to prevent users from providing undefined combinations of events.

Third, GUI-based programs are conservative in updating state. In particular, they avoid unnecessary updates, to suppress needlessly re-drawing widgets and notifying listeners. For instance, if an event wishes to set a widget to a state $\gamma$, then the event handler for that widget reads the current state of the widget, and does nothing if the current state is already $\gamma$, effectively treating the event as read-only.

Based on the above observations, we developed ACTEvE that does not extend event sequences that end in a readonly event. Pruning such sequences in a particular iteration of our approach prevents extensions of those sequences from being considered in future iterations, thereby providing compounded savings, while still ensuring completeness with respect to the AlLSEQS. We show that the read-only pattern is an instance of a more general notion of subsumption between event sequences. An event sequence that ends in a read-only event is subsumed by the same event sequence without that final event.

For our example music player app, using read-only subsumption, our approach explores 3,445 four-event sequences, compared to 21,117 by the ALLSEQS approach, which does not check for subsumption but is identical in all other respects. Besides clicks to many passive widgets in this app (e.g., LinearLayout) being read-only, another key reason for the savings is that even clicks to actionable widgets (e.g., Button) are read-only in many states of the app. For instance, consider the two-event sequence [stop, rewind]. The first event (stop) writes to many memory locations (e.g., fields mPlayer and $\mathrm{mState}$ in class MusicService shown in Figure 11). However, the second event (rewind) does not write to any location, because the processRewind() method of class MusicService that handles this event only writes if the state of the music player is Playing or Paused, whereas after the first stop event, its state is Stopped. Thus, our approach identifies the rewind event in sequence [stop, rewind] as read-only, and prunes all sequences explored by ALLSEQS that have this sequence as a proper prefix.

Section 4 presents a formal description of subsumption, the read-only instantiation of subsumption, and the formal guarantees it provides.

\section{GENERATING SINGLE EVENTS}

In this section, we describe how our approach systematically generates single events. We use tap events in Android as a proof-of-concept. Tap events are challenging to generate because they are continuous and have more variability than discrete events such as incoming phone calls, SMS messages, and battery charging events. Moreover, tap events are often the primary drivers of an app's functionality, and thus, control significantly more code of the app than other kinds of events. The principles underlying our approach, however, are not specific to tap events or to Android.

We begin by describing how Android handles tap events. Figure 4 shows the simplified code of the dispatchEvent () method of SDK class android.view.ViewGroup. When a tap event is input, this method is called recursively on wid-

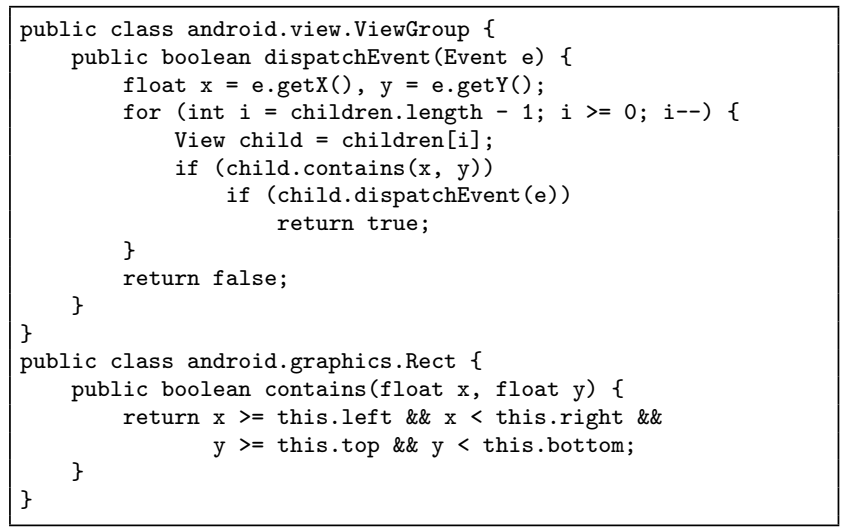

Figure 4: Source code snippet of Android SDK.

gets in the current screen's view hierarchy, to find the innermost widget to which to dispatch the event, starting with the root widget. If the event's coordinates lie within a widget's rectangle, as determined by the contains() method of SDK class android.graphics. Rect, then dispatchEvent() is called on children of that widget, from right to left, to determine whether the current widget is indeed the innermost one containing the event or if it has a descendant containing the event. For instance, any tap event that clicks on the pause button on the main screen of our example music player app results in testing for the event's containment in the following widgets in order, as the event is dispatched in the view hierarchy depicted in Figure 3 . We also indicate whether or not each test passes: FrameLayout ${ }^{1}$ (yes) $\rightarrow$ FrameLayout $^{2}$ (yes) $\rightarrow$ LinearLayout $^{2}$ (no) $\rightarrow$ LinearLayout $^{1}$ $($ yes $) \rightarrow$ Button $^{4}$ (no) $\rightarrow$ Button $^{3}$ (yes).

Our approach uses concolic testing to generate a separate tap event to each widget. This requires symbolically tracking events from the point where they originate to the point where they are handled. Let $(\$ x, \$ y)$ denote variables that our approach uses to symbolically track a concrete tap event $(x, y)$. Then, for each call to contains $(x, y)$ on a rectangle in the view hierarchy specified by constants $\left(x_{\text {left }}, x_{\text {right }}, y_{\text {top }}, y_{\text {bottom }}\right)$, our approach generates the following constraint or its negation, depending upon whether or not the tap event is contained in the rectangle:

$$
\left(x_{\text {left }} \leq \$ x<x_{\text {right }}\right) \wedge\left(y_{\text {top }} \leq \$ y<y_{\text {bottom }}\right)
$$

Our approach starts by sending a random tap event to the current screen of the given app. For our example app, suppose this event clicks the pause button. Then, our approach generates the following path constraint:

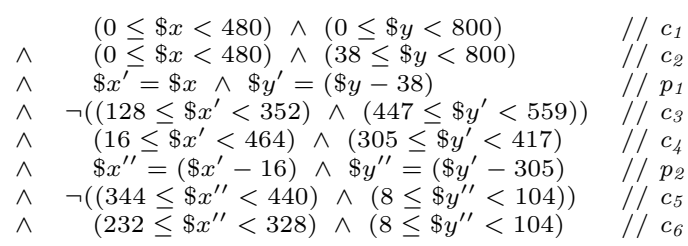

Constraints $c_{1}$ and $c_{2}$ capture the fact that the event is tested for containment in FrameLayout ${ }^{1}$ and FrameLayout ${ }^{2}$, respectively, and the test passes in both cases. The event is then tested against LinearLayout ${ }^{2}$ but the test fails (notice the negation in $c_{3}$ ). Constraints $c_{4}$ through $c_{6}$ arise from testing the event's containment in LinearLayout ${ }^{1}$, Button ${ }^{4}$ 
(the skip button), and Button ${ }^{3}$ (the pause button). We explain constraints $p_{1}$ and $p_{2}$ below.

Our approach next uses this path constraint to generate concrete tap events to other widgets. Specifically, for each $c_{i}$, it uses an off-the-shelf constraint solver to solve the constraint $\left(\bigwedge_{j=1}^{i-1} c_{j}\right) \wedge \neg c_{i}$ for $\$ x$ and $\$ y$. If this constraint is satisfiable, any solution the solver provides is a new concrete tap event guaranteed to take the path dictated by this constraint in the view hierarchy. That path in turn generates a new path constraint and our approach repeats the above process until all widgets in the hierarchy are covered.

We now explain the role of constraints $p_{1}$ and $p_{2}$ in the path constraint depicted above. These constraints introduce new symbolic variables $\$ x^{\prime}, \$ y^{\prime}, \$ x^{\prime \prime}$, and $\$ y^{\prime \prime}$. They arise because, as a tap event is dispatched in the Android SDK, various offsets are added to its concrete $x$ and $y$ coordinates, to account for margins, convert from relative to absolute positions, etc. The already-simplified path constraint depicted above highlights the complexity for concolic execution that a real platform like Android demands: we instrument not only the SDK code shown in Figure 4 but all SDK code, as well as the code of each app under test. Dropping any of the above constraints due to missed instrumentation can result in the path divergence problem [15] in concolic testing, where the concrete and symbolic values diverge and threaten the ability to cover all widgets.

\section{GENERATING EVENT SEQUENCES}

In this section, we describe how our approach generates sequences of events. To specify our approach fully and to express and prove the formal guarantee of the approach, we use a simple imperative language, which includes the essential features of Android apps. We begin with the explanation of our language and the associated key semantic concepts (Sections 4.1 and 4.2). We then describe our algorithm, proceeding from the top-level routine (Section 4.3) to the main optimization operator (Sections 4.4 and 4.5). Finally, we discuss the formal completeness guarantee of our algorithm (Section 4.6). (Appendix B presents the proofs of all lemmas and the theorem discussed in this section.)

\subsection{Core Language}

Our programming language is a standard WHILE language with one fixed input variable $a$ and multiple global variables $g_{1}, \ldots, g_{m}$ for some fixed $m$. A program $s$ models an Android app, and it is meant to run repeatedly in response to a sequence of input events provided by an external environment, such as a user of the app. The global variables are threaded in the repetition, so that the final values of these variables in the $i$-th iteration become the initial values of the variables in the following $(i+1)$-th iteration. In contrast, the input variable $a$ is not threaded, and its value in the $i$-th iteration comes from the $i$-th input event. Other than this initialization in each iteration, no statements in the program $s$ can modify the input variable $a$.

The syntax of the language appears in Figure 5 For simplicity, the language assumes that all the input events are given by integers and stored in the input variable $a$. It allows such an event in $a$ to participate in constructing complex expressions $e$, together with global variable $g$ and the application of an arithmetic operator aop $(\bar{e})$, such as $a+g$ and 3. Boolean expressions $b$ combine the expressions using standard comparison operators, such as $=$ and $\leq$, and

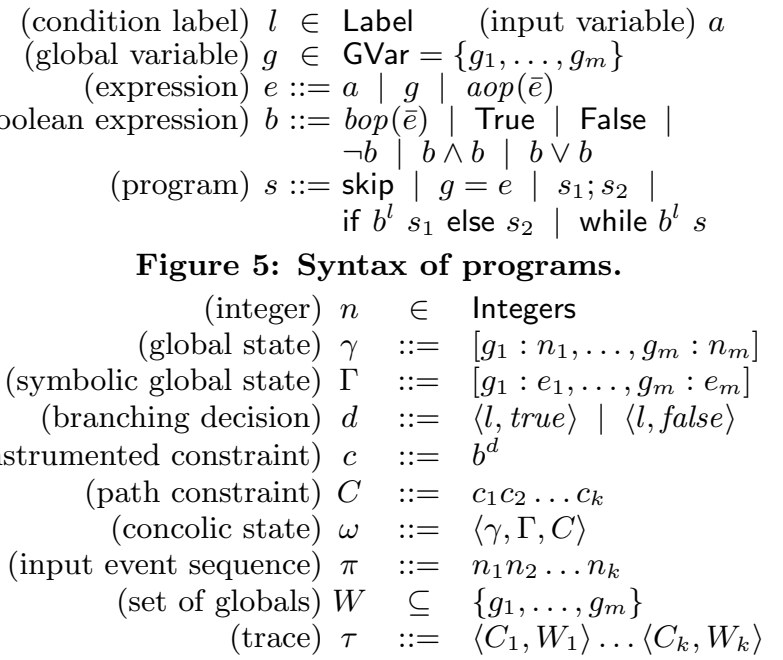

Figure 6: Semantic domains.

build conditions on program states. Our language allows five types of programming constructs with the usual semantics: skip for doing nothing; assignments to global variables $g=e$; sequential compositions $\left(s_{1} ; s_{2}\right)$; conditional statements (if $b^{l} s_{1}$ else $s_{2}$ ) with an $l$-labeled boolean $b$; and loops (while $b^{l} s$ ). Note that although the input variable $a$ can appear on the RHS of an assignment, it is forbidden to occur on the LHS. Thus, once initialized, the value of $a$ never changes during the execution of a program. Note also that all boolean conditions are annotated with labels $l$. We require the uniqueness of these labels. The labels will be used later to track branches taken during the execution of a program.

Example 1. The following program is a simplified version of the music player app in our language:

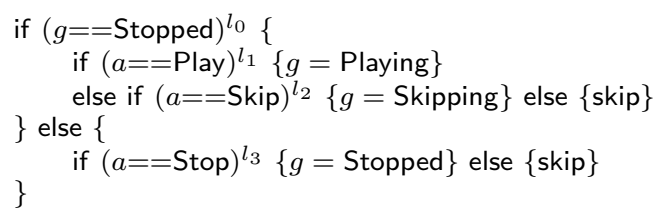

To improve the readability, we use macros here: Stopped = Stop $=0$, Playing $=$ Play $=1$, and Skipping $=$ Skip $=2$. Initially, the player is in the Stopped state (which is the value stored in $g$ ), but it can change to the Playing or Skipping state in response to an input event. When the player gets the Stop input event, the player's state goes back to Stopped.

We write Globals $(e)$ and Globals $(s)$ to denote the set of free global variables appearing in $e$ and $s$, respectively.

Throughout the rest of the paper, we fix the input program and the initial global state given to our algorithm, and denote them by $s_{i n}$ and $\gamma_{i n}$.

\subsection{Semantic Domains}

We interpret programs using a slightly non-standard operational semantics, which describes the concolic execution of a program, that is, the simultaneous concrete and symbolic execution of the program. Figure 6 summarizes the major semantic domains. The most important are those for concolic states $\omega$, input sequences $\pi$, and traces $\tau$.

A concolic state $\omega$ specifies the status of global variables concretely as well as symbolically. The state consists of the 
three components, denoted by $\omega \cdot \gamma, \omega \cdot \Gamma$, and $\omega \cdot C$, respectively. The $\gamma$ component keeps the concrete values of all the global variables, while the $\Gamma$ component stores the symbolic values of them, specified in terms of expressions. We require that global variables do not occur in these symbolic values; only the input variable $a$ is allowed to appear there. The $C$ component is a sequence of instrumented constraints $c_{1} c_{2} \ldots c_{k}$, where each $c_{i}$ is a boolean expression $b$ annotated with a label and a boolean value. As for symbolic values, we prohibit global variables from occurring in $b$. The annotation indicates the branch that generates this boolean value as well as the branching decision observed during the execution of a program.

An input event sequence $\pi$ is just a finite sequence of integers, where each integer represents an input event from the environment.

A trace $\tau$ is also a finite sequence, but its element consists of a path constraint $C$ and a subset $W$ of global variables. The element $\langle C, W\rangle$ of $\tau$ expresses what happened during the concolic execution of a program with a single input event (as opposed to an event sequence). Hence, if $\tau$ is of length $k$, it keeps the information about event sequences of length $k$. The $C$ part describes the symbolic path constraint collected during the concolic execution for a single event, and the $W$ part stores variables written during the execution. As in the case of concolic state, we adopt the record selection notation, and write $\left(\tau_{i}\right) \cdot C$ and $\left(\tau_{i}\right) . W$ for the $C$ and $W$ components of the $i$-th element of $\tau$. Also, we write $\tau\langle C, W\rangle$ to mean the concatenation of $\tau$ with a singleton trace $\langle C, W\rangle$.

Our operational semantics defines two evaluation relations: $(1)\langle s, n, \omega\rangle \downarrow \omega^{\prime} \triangleright W$ and $(2)\langle s, \pi, \gamma\rangle \Downarrow \gamma^{\prime} \triangleright \tau$. The first relation models the run of $s$ with a single input event $n$ from a concolic initial state $\omega$. It says that the outcome of this execution is $\omega^{\prime}$, and that during the execution, variables in $W$ are written. Note that the path constraint $\omega^{\prime}$.C records all the branches taken during the execution of a program. If the execution encounters a boolean condition $b^{l}$ that evaluates to True, it still adds True ${ }^{\langle l, t r u e\rangle}$ to the $C$ part of the current concolic state, and remembers that the true branch is taken. The case that $b^{l}$ evaluates to False is handled similarly.

The second relation describes the execution of $s$ with an input event sequence. It says that if a program $s$ is run repeatedly for an input sequence $\pi$ starting from a global state $\gamma$, this execution produces a final state $\gamma^{\prime}$, and generates a trace $\tau$, which records path constraints and written variables during the execution. Note that while the first relation uses concolic states to trace various symbolic information about execution, the second uses traces for the same purpose.

The rules for the evaluation relations mostly follow from our intended reading of all the parts in the relations. They are described in detail in Appendix A.

Recall that we fixed the input program and the initial global state and decided to denote them by $s_{i n}$ and $\gamma_{i n}$. We say that a trace $\tau$ is feasible if $\tau$ can be generated by running $s_{i n}$ from $\gamma_{i n}$ with some event sequences, that is,

$$
\exists \pi, \gamma^{\prime} .\left\langle s_{i n}, \pi, \gamma_{i n}\right\rangle \Downarrow \gamma^{\prime} \triangleright \tau .
$$

Our algorithm works on feasible traces, as we explain next.

\subsection{Algorithm}

ACTEve takes a program, an initial global state, and an upper bound $k$ on the length of event sequences to explore.

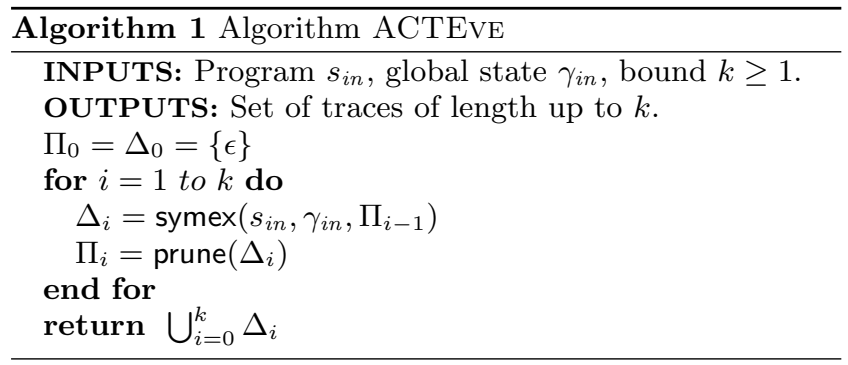

By our convention, $s_{i n}$ and $\gamma_{i n}$ denote these program and global state. Then, ACTEvE generates a set $\Sigma$ of feasible traces of length up to $k$, which represents event sequences up to $k$ that achieve the desired code coverage.

Formally, the output $\Sigma$ of our algorithm satisfies two correctness conditions.

1. First, all traces in $\Sigma$ are feasible. Every $\tau \in \Sigma$ can be generated by running $s_{i n}$ with some event sequence $\pi$ of length up to $k$.

2. Second, $\Sigma$ achieves the full coverage in the sense that if a branch of $s_{i n}$ is covered by an event sequence $\pi$ of length up to $k$, we can find a trace $\tau$ in $\Sigma$ such that every event sequence $\pi^{\prime}$ satisfying $\tau$ (i.e., $\pi^{\prime} \mid=\tau$ ) also covers the branch.

The top-level routine of ACTEvE is given in Algorithm 1 The routine repeatedly applies operations symex and prune in alternation on sets $\Pi_{i}$ and $\Delta_{i}$ of traces of length $i$, starting with the set containing only the empty sequence $\epsilon$. Figure 7 illustrates this iteration process pictorially. The iteration continues until a given bound $k$ is reached, at which point $\bigcup_{i=1}^{k} \Delta_{i}$ is returned as the result of the routine.

The main work of ACTEVE is done mostly by the operations symex and prune. It invokes $\operatorname{symex}\left(s_{i n}, \gamma_{i n}, \Pi_{i-1}\right)$ to generate all the feasible one-step extensions of traces in $\Pi_{i-1}$. Hence,

$$
\begin{aligned}
& \underset{\operatorname{symex}}{\sin }\left(s_{i n}, \gamma_{i n}, \Pi_{i-1}\right)= \\
& \quad\left\{\tau\langle C, W\rangle \mid \tau \in \Pi_{i-1} \text { and } \tau\langle C, W\rangle \text { is feasible }\right\},
\end{aligned}
$$

where $\tau\langle C, W\rangle$ means the concatenation of $\tau$ with a singlestep trace $\langle C, W\rangle$. The symex operation can be easily implemented following a standard algorithm for concolic execution (modulo the well-known issue with loops), as we did in our implementation for Android ${ }^{2}$ In fact, if we skip the pruning step in ACTEvE and set $\Pi_{i}$ to $\Sigma_{i}$ there (equivalently, prune $(\Delta)$ returns simply $\Delta$ ), we get the standard concolic execution algorithm, AlLSEQS for exploring all branches that are reachable by event sequences of length $k$ or less.

The goal of the other operation prune is to identify traces that can be thrown away without making the algorithm cover less branches, and to filter out such traces. This filtering is the main optimization employed in our algorithm. It is based on our novel idea of subsumption between traces, which we discuss in detail in the next subsection.

Example 2. We illustrate our algorithm with the music player app in Example 1 and the bound $k=2$. Initially, the algorithm sets $\Pi_{0}=\Delta_{0}=\{\epsilon\}$. Then, it extends this empty

\footnotetext{
${ }^{2}$ When $s_{i n}$ contains loops, the standard concolic execution can fail to terminate. However, symex is well-defined for such programs, because it is not an algorithm but a declarative specification.
} 


$$
\{\epsilon\}=\Pi_{0} \stackrel{\text { symex }}{\longrightarrow} \Delta_{1} \stackrel{\text { prune }}{\longrightarrow} \Pi_{1} \stackrel{\text { symex }}{\longrightarrow} \Delta_{2} \stackrel{\text { prune }}{\longrightarrow} \Pi_{2} \stackrel{\text { symex }}{\longrightarrow} \ldots
$$

Figure 7: Simulation of our ACTEVE algorithm.

sequence by calling symex, and obtains $\Delta_{1}$ that contains the following three traces of length 1 :

$$
\begin{aligned}
& \tau=\left\langle\text { True }^{\left\langle l_{0}, \text { true }\right\rangle}(a==\text { Play })^{\left\langle l_{1}, \text { true }\right\rangle},\{g\}\right\rangle, \\
& \tau^{\prime}=\left\langle\text { True }^{\left\langle l_{0}, \text { true }\right\rangle}(a==\text { Play })^{\left\langle l_{1}, \text { false }\right\rangle}(a==\text { Skip })^{\left\langle l_{2}, \text { true }\right\rangle},\{g\}\right\rangle, \\
& \tau^{\prime \prime}=\left\langle\text { True }^{\left\langle l_{0}, \text { true }\right\rangle}(a==\text { Play })^{\left\langle l_{1}, \text { false }\right\rangle}(a==\text { Skip })^{\left\langle l_{2}, \text { false }\right\rangle}, \emptyset\right\rangle .
\end{aligned}
$$

Trace $\tau$ describes the execution that takes the true branches of $l_{0}$ and $l_{1}$. It also records that variable $g$ is updated in this execution. Traces $\tau^{\prime}$ and $\tau^{\prime \prime}$ similarly correspond to executions that take different paths through the program.

Next, the algorithm prunes redundant traces from $\Delta_{1}$. It decides that $\tau^{\prime \prime}$ is such a trace, filters $\tau^{\prime \prime}$, and sets $\Pi_{1}=$ $\left\{\tau, \tau^{\prime}\right\}$. This filtering decision is based on the fact that the last step of $\tau^{\prime \prime}$ does not modify any global variables. For now, we advise the reader not to worry about the justification of this filtering; it will be discussed in the following subsections.

Once $\Delta_{1}$ and $\Pi_{1}$ are computed, the algorithm goes to the next iteration, and computes $\Delta_{2}$ and $\Pi_{2}$ similarly. The trace set $\Delta_{2}$ is obtained by calling symex, which extends traces in $\Pi_{1}$ with one further step:

$$
\begin{aligned}
\Delta_{2}=\{ & \tau\left\langle\text { True }^{\left\langle l_{0}, \text { false }\right\rangle}(a==\text { Stop })^{\left\langle l_{3}, \text { true }\right\rangle},\{g\}\right\rangle, \\
& \tau\left\langle\text { True }^{\left\langle l_{0}, \text { false }\right\rangle}(a==\text { Stop })^{\left\langle l_{3}, \text { false }\right\rangle}, \emptyset\right\rangle, \\
& \tau^{\prime}\left\langle\text { True }^{\left\langle l_{0}, \text { false }\right\rangle}(a==\text { Stop })^{\left\langle l_{3}, \text { true }\right\rangle},\{g\}\right\rangle, \\
& \left.\tau^{\prime}\left\langle\text { True }^{\left\langle l_{0}, \text { false }\right\rangle}(a==\text { Stop })^{\left\langle l_{3}, \text { false }\right\rangle}, \emptyset\right\rangle\right\}
\end{aligned}
$$

Among these traces, only the first and the third have the last step with the nonempty write set, so they survive pruning and form the set $\Pi_{2}$.

After these two iterations, our algorithm returns $\bigcup_{i=0}^{2} \Delta_{i}$.

\subsection{Subsumption}

For a feasible trace $\tau$, we define

$$
\operatorname{final}(\tau)=\left\{\gamma^{\prime} \mid \exists \pi .\left\langle s_{i n}, \pi, \gamma_{i n}\right\rangle \Downarrow \gamma^{\prime} \triangleright \tau\right\},
$$

which consists of the final states of the executions of $s_{i n}$ that generate the trace $\tau$.

Let $\tau$ and $\tau^{\prime}$ be feasible traces. The trace $\tau$ is subsumed by $\tau^{\prime}$, denoted $\tau \sqsubseteq \tau^{\prime}$, if and only if final $(\tau) \subseteq$ final $\left(\tau^{\prime}\right)$. Note that the subsumption compares two traces purely based on their final states, ignoring other information like length or accessed global variables. Hence, the subsumption is appropriate for comparing traces when the traces are used to represent sets of global states, as in our algorithm ACTEvE. We lift subsumption on sets $T, T^{\prime}$ of feasible traces in a standard way: $T \sqsubseteq T^{\prime} \Longleftrightarrow \forall \tau \in T . \exists \tau^{\prime} \in T^{\prime} . \tau \sqsubseteq \tau^{\prime}$. Both the original and the lifted subsumption relations are preorder, i.e., they are reflexive and transitive.

A typical use of subsumption is to replace a trace set $T_{\text {new }}$ by a subset $T_{o p t}$ such that $T_{n e w} \sqsubseteq T_{o p t} \cup T_{\text {old }}$ for some $T_{\text {old }}$. In this usage scenario, $T_{n e w}$ represents a set of traces that a concolic testing algorithm originally intends to extend, and $T_{\text {old }}$ that of traces that the algorithm has already extended. Reducing $T_{\text {new }}$ to $T_{\text {opt }}$ entails that fewer traces will be explored, so it boosts the performance of the algorithm.
Why is it ok to reduce $T_{\text {new }}$ to $T_{\text {opt }}$ ? An answer to this question lies in two important properties of the subsumption relation. First, the symex operation preserves the subsumption relationship.

Lemma 1. For sets $T, T^{\prime}$ of feasible traces,

$$
T \sqsubseteq T^{\prime} \Longrightarrow \operatorname{symex}\left(s_{i n}, \gamma_{i n}, T\right) \sqsubseteq \operatorname{symex}\left(s_{i n}, \gamma_{i n}, T^{\prime}\right) \text {. }
$$

Second, if $T$ is subsumed by $T^{\prime}$, running symex with $T^{\prime}$ will cover as many branches as what doing the same thing with $T$ covers. Let

$\operatorname{branch}(C)=\left\{\langle l, v\rangle \mid b^{\langle l, v\rangle}=C_{i}\right.$ for some $\left.i \in\{1, \ldots,|C|\}\right\}$.

The formal statement of this second property appears in the following lemma:

Lemma 2. For all sets $T, T^{\prime}$ of feasible traces, if $T \sqsubseteq T^{\prime}$.

$\bigcup\left\{\operatorname{branch}\left(\left(\tau_{|\tau|}\right) \cdot C\right) \mid \tau \in \operatorname{symex}\left(s_{i n}, \gamma_{i n}, T\right)\right\}$ $\subseteq \bigcup\left\{\operatorname{branch}\left(\left(\tau_{|\tau|}\right) . C\right) \mid \tau \in \operatorname{symex}\left(s_{i n}, \gamma_{i n}, T^{\prime}\right)\right\}$.

In the lemma, $\tau_{|\tau|}$ means the last element in the trace $\tau$ and $\left(\tau_{|\tau|}\right) . C$ chooses the $C$ component of this element. So, the condition compares the branches covered by the last elements of traces.

Using these two lemmas, we can now answer our original question about the subsumption-based optimization. Suppose that $T_{\text {opt }}$ is a subset of $T_{\text {new }}$ but $T_{\text {new }} \sqsubseteq T_{\text {opt }} \cup T_{\text {old }}$ for some $T_{\text {old }}$. The lemmas imply that every new branch covered by extending $T_{\text {new }}$ for the further $k \geq 1$ steps is also covered by doing the same thing for $T_{\text {opt }} \cup T_{\text {old }}$. More concretely, according to Lemma 1 , the extension of $T_{\text {new }}$ for the further $k-1$ or smaller steps will continue to be $\sqsubseteq$-related to that of $T_{\text {opt }} \cup T_{\text {old }}$. Hence, running symex with such extended $T_{\text {new }}$ will cover only those branches that can also be covered by doing the same thing for the similarly extended $T_{\text {opt }} \cup T_{\text {old }}$ (Lemma 2). Since we assume that the $k$ or smaller extensions of $T_{\text {old }}$ are already explored, this consequence of the lemmas mean that as long as we care about only newly covered branches, we can safely replace $T_{n e w}$ by $T_{o p t}$, even when $T_{\text {opt }}$ is a subset of $T_{\text {new }}$.

\subsection{Pruning}

The goal of the pruning operator is to reduce a set $\Delta$ of feasible traces to a subset $\Pi \subseteq \Delta$, such that $\Delta$ is subsumed by $\Pi$ and all strict prefixes of $\Delta\}^{3}$

$$
\Delta \sqsubseteq \Pi \cup \operatorname{sprefix}(\Delta),
$$

where $\operatorname{sprefix}(\Delta)=\left\{\tau\left|\exists \tau^{\prime}.\right| \tau^{\prime} \mid \geq 1 \wedge \tau \tau^{\prime} \in \Delta\right\}$. The reduction brings the gain in performance, while the subsumption relationship (together with an invariant maintained by ACTEve) ensures that no branches would be missed by this optimization.

Our implementation of pruning adopts a simple strategy for achieving the goal. From a given set $\Delta$, the operator filters out all traces whose last step does not involve any writes, and returns the set $\Pi$ of remaining traces. The implementation appears in Figure 2, and accomplishes our goal, as stated in the following lemma:

Lemma 3. For all sets $\Delta$ of feasible traces, $\operatorname{rprune}(\Delta)$ is a subset of $\Delta$ and satisfies the condition in (1).

\footnotetext{
${ }^{3}$ This condition typechecks because all prefixes of feasible traces are again feasible traces so that the RHS of $\sqsubseteq$ contains only feasible traces.
} 


\begin{tabular}{l}
\hline Algorithm 2 The rprune operation \\
INPUTS: Set $\Delta$ of traces. \\
OUTPUTS: Set $\Pi=\left\{\tau|\tau \in \Delta \wedge| \tau \mid \geq 1 \wedge\left(\tau_{|\tau|}\right) . W=\emptyset\right\}$ \\
\hline
\end{tabular}

Note that the pruning operator can be implemented differently from rprune. As long as the pruned set $\Pi$ satisfies the subsumption condition in (1), our entire algorithm ACTEvE remains relatively complete, meaning that the optimization with pruning will not introduce new uncovered branches. In Appendix $\mathrm{C}$, we include another implementation of pruning that uses the notion of independence.

\subsection{Relative Completeness}

For $i \geq 0$, let symex ${ }^{i}\left(s_{i n}, \gamma_{i n}, T\right)$ be the $i$-repeated application of symex $\left(s_{i n}, \gamma_{i n},-\right)$ to a set $T$ of feasible traces, where the 0 -repeated application symex ${ }^{0}\left(s_{i n}, \gamma_{i n}, T\right)$ is defined to be $T$. Also, lift the branch operation to a trace set:

$$
\operatorname{branch}(T)=\bigcup\left\{\operatorname{branch}\left(\left(\tau_{i}\right) . C\right) \mid \tau \in T \wedge i \in\{1, \ldots,|\tau|\}\right\}
$$

Theorem 4 (Completeness). For every $k \geq 1$,

$$
\begin{aligned}
& \operatorname{branch}\left(\operatorname{ACTEVE}\left(s_{i n}, \gamma_{i n}, k\right)\right) \\
& =\operatorname{branch}\left(\bigcup_{i=0}^{k} \operatorname{symex}^{i}\left(s_{i n}, \gamma_{i n},\{\epsilon\}\right)\right) .
\end{aligned}
$$

The RHS of the equation in the theorem represents branches covered by running the standard concolic execution without pruning. The theorem says that our algorithm covers the same set of branches, hence same program statements, as the standard concolic execution.

\section{EMPIRICAL EVALUATION}

In this section, we present the empirical evaluation of our technique. First, we describe the implementation of ACTEve (Section 5.1). Next, we present the studies, including the subjects used, the empirical setup, and the study results (Sections 5.2 5.4). Finally, we discuss threats to the validity of the studies (Section 5.5).

\subsection{Implementation}

The implementation of our system uses the Soot framework 31, and consists of 11,000 lines of Java code. Figure 8 shows a dataflow diagram of our system. Our system inputs Java bytecodes of classes in Android SDK and App under test. Java bytecodes of a class are obtained by either compiling its source code (as done in our empirical evaluation) or converting Android's Dalvik bytecode into Java bytecodes 24]. Our system outputs a set of tests, Test inputs, each of which denotes an event sequence. The script shown in the inset box is an example of a test that our system can generate.

Each line in the script corresponds to an event. Tap generates a tap event on the screen at the specified

$\mathrm{X}$ and $\mathrm{Y}$ coordinates. Tests similar to the one in this script can be automatically executed using Monkey - a tool in the Android software development kit.

Our system consists of four components: Instrumenter, Runner, Concolic testing engine, and Subsumption analyzer. We explain each of them in turn.

Instrumenter inputs Android $S D K$, and the Java class files of the App under test, and outputs Instrumented ( $S D K+A p p)$.
This component instruments the Java bytecodes of each class of the App under test and any third-party libraries that the $A p p$ uses. It also instruments classes in the Android framework (e.g., android.*) but this step is performed only once because the way in which a class is instrumented does not depend on any other class.

Instrumenter operates on a three-address form of Java bytecode produced by Soot, called Jimple. Instrumenter performs three types of instrumentations. First, it instruments $A p p$ for concolic execution, which involves two main steps: (1) adds a meta variable (field) that stores the symbolic value corresponding to each variable (field); (2) inserts a new assignment before every assignment such that the new assignment copies the content of meta variable (field) corresponding to the $\mathrm{r}$-value of the original assignment to the meta variable (field) corresponding to l-value of the original assignment. Second, Instrumenter instruments $A p p$ to record fields of Java classes that are written only during the app responds to the last event in the sequence of events corresponding to a test. Third, Instrumenter ensures that in Instrumented $(S D K+A p p)$, user-specified method summaries are symbolically executed instead of the original methods.

Runner inputs Instrumented ( $S D K+A p p)$. The first time the component is called, it generates a test randomly; thereafter, it inputs tests from either the Concolic testing engine or the Subsumption analyzer. Runner outputs Test inputs that includes the randomly-generated test and tests that it inputs. For each of those tests in Test inputs, it also outputs a Path constraint and a Write set, which are used internally by the other two components.

Runner executes Instrumented ( $A p p$ ) with the test on an emulator that uses Instrumented ( $S D K$ ). Besides these Android framework classes, no other components of the framework, such as Dalvik virtual machine of the Android execution environment, are modified. This feature of our system makes it easily portable to different versions of Android.

Execution of a test generates the path constraint of the path that the App takes and Write set, which is a set of fields of Java classes that are written during the last event in the input event sequence. Writes to array elements are recorded as writes to one distinguished field. Runner uses a set of (typically 16) emulators each of which can execute a different test at any time. Such parallelism enables our system to perform systematic testing of realistic apps. Execution of an app in a realistic environment, such as an emulator or an actual device, takes orders of magnitude more time than execution of similar desktop applications.

Concolic testing engine inputs Path constraint of a path, and outputs New tests for current iteration. The component first computes a set of new path constraints by systematically negating each atomic constraint (i.e., conjunct) of the input path constraint, as in standard concolic testing. Then, it checks satisfiability of each of those new path constraints, and generates and outputs new tests corresponding to satisfiable path constraints using the Z3 SMT solver [7].

Subsumption analyzer inputs Write set, a set of fields of Java classes that are written when $A p p$ responds to the last event in the event sequence corresponding to a specific test. It may output one Seed test for next iteration.

Subsumption analyzer implements the rprune operator in Algorithm 2. It outputs the test that corresponds to its input Write set if Write set is non-empty. The output test 


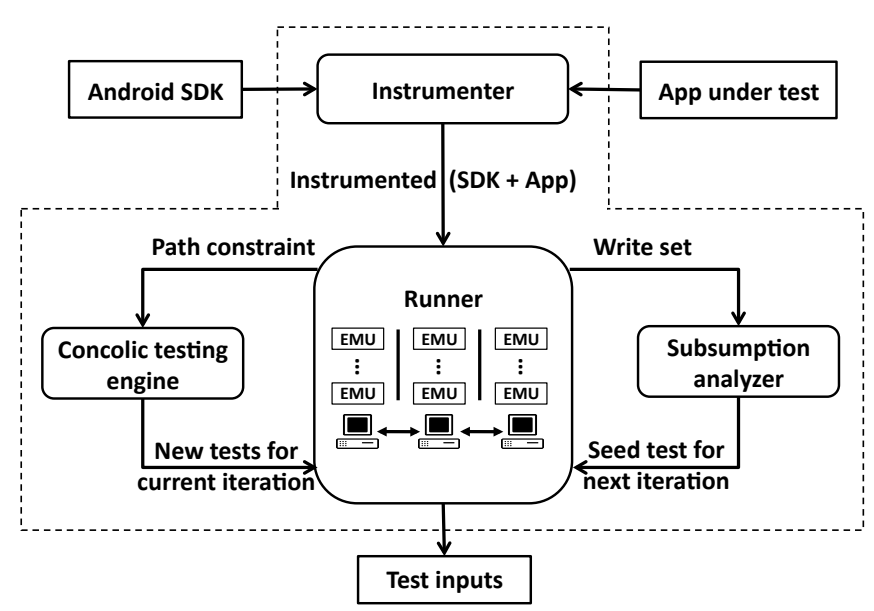

Figure 8: Dataflow diagram of our system.

is called the seed test because new tests are generated in the next iteration by extending this test with new events. If Write set is empty, Subsumption analyzer outputs no test.

One important feature of Subsumption analyzer is that it can be configured to ignore writes to a given set of fields. This feature is useful because, in Android, many events lead to writes to some memory locations, which fall into two classes: (1) locations that are written to and read from during the same event of an event sequence (i.e., never written and read across events); (2) locations that result from Android's low-level operations, such as optimizing performance and memory allocation, and correspond to fields of Android classes that are irrelevant to an app's behavior. Subsumption analyzer ignores writes to these two classes of writes because they are irrelevant to an app's behavior in subsequent events of an event sequence.

\subsection{Subject Apps}

We used five open-source Android apps for our studies. Random Music Player (RMP) is the app that is used as the example in Section 2 Sprite is an app for comparing the relative speeds of various $2 \mathrm{D}$ drawing methods on Android. Translate is an app for translating text from one language to another using Google's Translation service on the Web. Timer is an app for providing a countdown timer that plays an alarm when it reaches zero. Ringdroid is an app for recording and editing ring tones.

\subsection{Study 1}

The goal of this study is to measure the improvement in efficiency of ACTEve over AllSeqs. First, we performed concolic execution for each subject using ACTEvE and AllSEQS. We used $k=4$ for RMP, Translate, and Sprite. However, because AlLSEQS did not terminate for the other two apps when $k=4$ in the 12 -hour time limit set for experiments, we used $k=3$ for Timer and $k=2$ for Ringdroid. Note that ACTEve terminated for all five apps even for $k=4$. In this step, we used 16 concurrently running emulators to execute tests and compute path constraints for corresponding program paths. Second, for each algorithm, we computed three metrics:

1. The running time of the algorithm.

2. The number of feasible paths that the algorithm finds.

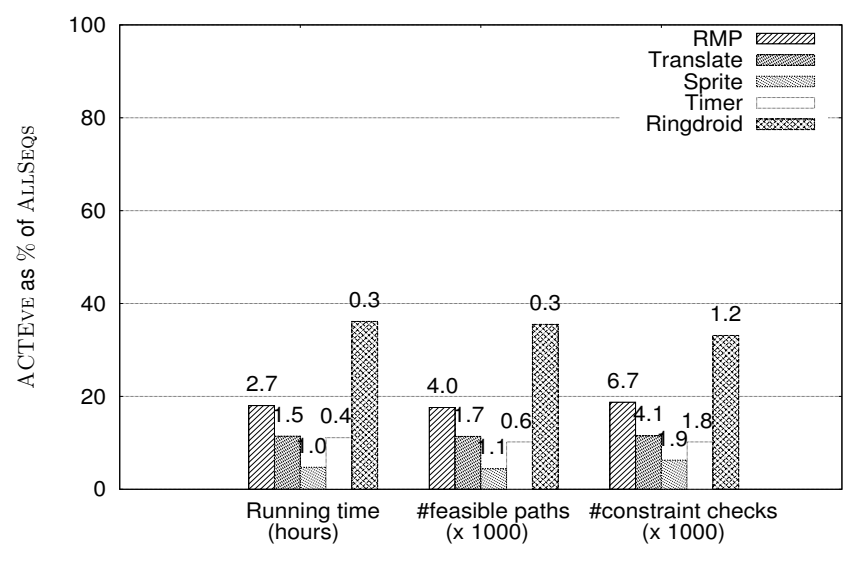

Figure 9: Results of Study 1: Running time, number of feasible paths explored, and number of constraint checks made by ACTEVE normalized with respect to those metrics for ALLSEQS.

3. The number of satisfiability checks of path constraints that the algorithm makes.

We measure the running time of the algorithms (metric (1)) because comparing them lets us determine the efficiency of ACTEve over AllSEqs. However, by considering only running time, it may be difficult to determine whether the efficiency of our algorithm will generalize to other application domains and experimental setups. Furthermore, we need to verify that the savings in running time is due to the reduction provided by our algorithm. Thus, we also compute the other two metrics (metrics (2) and (3)).

Figure 9 shows the results of the study for our subjects. In the figure, the horizontal axis represents the three metrics, where each cluster of bars corresponds to one metric. Within each cluster, the five bars represent the corresponding metric for the five subjects. In the first cluster, the height of each bar represents the normalized ratio (expressed as a percentage) of the running time of ACTEVE to that of ALLSEQS. The number at the top of each bar in this cluster is the running time of ACTEve measured in hours. Similarly, in the second and third clusters, the height of each bar represents the normalized ratio of the number of feasible paths explored and the number of constraint checks made, respectively, by ACTEve to the corresponding entities for AllSeqs. The number at the top of each bar in the second and third clusters is the number of feasible paths explored and the number of constraint checks made, respectively, by ACTEvE. For brevity, these numbers are rounded, and shown as multiples of a thousand. For example, the first cluster shows the ratio of the running time of ACTEVE to that of AlLSEQS: RMP is $18 \%$; Translate is $15 \%$; Sprite is $5 \%$; Timer is $11 \%$; Ringdroid is $36 \%$. This cluster also shows that the running time of ACTEve is 2.7 hours for RMP, 1.5 hours for Translate, 1 hour for Sprite, 0.4 hours for Timer, and 0.3 hours for Ringdroid.

The results of the study show that ACTEvE is significantly more efficient than AlLSEQS. ACTEvE requires only a small fraction (5\%-36\%) of the running time of ALLSEQS to achieve the same completeness guarantee. Thus, using ACTEve provides significant savings in running time over AllSEQS (64\%-95\%). The results also illustrate why the running time for ACTEVE is significantly less than for ALLSEQS: 

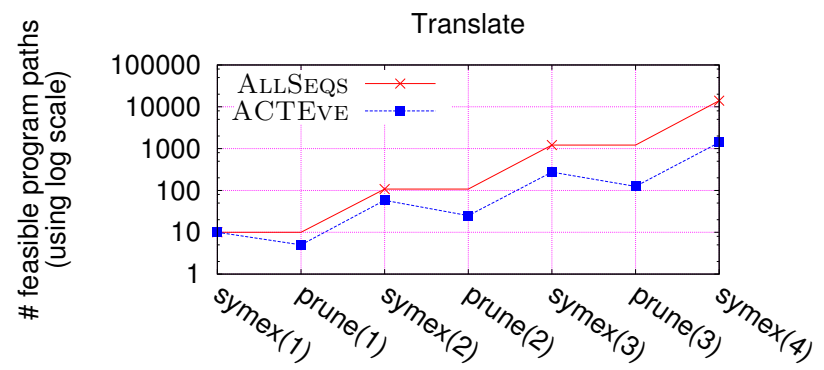

Figure 10: Results of Study 2 for translate app: The number of paths (using a logarithmic scale) after symex and prune operations in each iteration.

ACTEvE explores only $4.4 \%-35.5 \%$ of all feasible paths that AlLSEQS explores; ACTEVE checks significantly fewer constraints $(6.2 \%-33.1 \%)$ than AlLSEQS.

\subsection{Study 2}

The goal of this study is to record the number of paths pruned by ACTEVE because this reduction in the number of paths explored highlights why ACTEvE is more efficient than AllSeQs. To do this, we performed concolic execution of each app for $k=4$, and we recorded the following information for each iteration of ACTEvE and ALLSEQS:

1. The number of feasible paths that symex explores; recall that symex explores new feasible paths.

2. The number of feasible paths that remain after prune.

Figure 10 shows the results of the study for one subject app; the results for the remaining apps are similar, and are shown in Appendix D. In each graph, the horizontal axis represents the symex and prune operations performed in each iteration. The vertical axis shows the number of paths using a log scale. For example, the graph for Translate in Figure 10 shows that ACTEvE explores 274 paths in iteration 3. The subsequent pruning step filters out 149 paths. Thus, only the remaining 125 paths are extended in iteration 4 . In contrast, AllSEQS explores 1,216 paths in iteration 3, all of which are extended in iteration 4 .

The results clearly show the improvement achieved by the pruning that ACTEvE performs. First, the graphs show that ACTEve explores many fewer paths than ALLSEQS, and the rate of improvement increases as the number of iterations increases. For example, in the fourth iteration of symex, ACTEve explores 1,402 paths and ALLSEQs has explored 13,976 paths. Second, the graphs also show that, at each iteration of prune, the number of paths that will then be extended decreases: the descending line in the graphs represents the savings that prune produces. In contrast, the horizontal line for the same interval corresponding to ALLSEQS shows that no pruning is being performed.

\subsection{Threats to Validity}

There are several threats to the validity of our studies. The main threat to internal validity arises because our system is configured to ignore writes to certain fields that do not affect an app's behavior (see Section 5.1 under "Subsumption analyzer"). We mitigate this threat in two ways. First, our implementation ignores only fields of Android's internal classes that are clearly irrelevant to an app's behavior; it never ignores fields of app classes, third-party libraries, or fields of Android classes (e.g., widgets) that store values that can be read by an app. Second, we ran our system using the ALLSEQS algorithm (that performs no pruning), and checked if any ignored field is written in one event and read in a later event of an event sequence. Most of the fields that our system is configured to ignore are never read and written across events. For the few that were, we manually confirmed that it is safe to ignore them.

Threats to external validity arise when the results of the experiment cannot be generalized. We evaluated our technique with only five apps. Thus, the efficiency of our technique may vary for other apps. However, our apps are representative of typical Android apps considering the problem that our technique addresses.

\section{RELATED WORK}

Memon 20 presented the first framework for generating, running, and evaluating GUI tests. Several papers (e.g., 44, 22, 23, 34, ) present components and extensions of this framework. Most existing GUI testing approaches either use capture-replay to infer the GUI model automatically [21, 30] or require users to provide the GUI model 33, 35. An exception is the work of Ganov et al. 10, which uses symbolic execution to infer data inputs to GUI components. Our work also uses symbolic execution but focuses on event inputs. Our techniques for efficiently generating sequences of events are complementary to the above approaches.

Significant advances have been made in recent years to alleviate path explosion in concolic testing. These include compositional testing (e.g., [1, 11]), using program dependence information to avoid analyzing redundant paths (e.g., 3 19, 26, 27]), using input grammars (e.g., 13]), using models of library classes (e.g., 17]) or constraint solvers that support higher-level program abstractions (e.g, 2, 32]), and using path-exploration heuristics that cover deep internal parts of a program (e.g., 18, 25]). Our input subsumption idea is complementary to the above ideas for taming path explosion. Our system indeed leverages some of the above ideas. It uses (1) method summaries and models for certain Android framework classes, (2) a grammar to specify input events, and (3) state-of-the-art constraint solving provided by the Z3 SMT solver.

\section{CONCLUSION AND FUTURE WORK}

In this paper, we presented a technique, ACTEvE to automatically and systematically generate input events to exercise smartphone apps. We described our system that implements ACTEVE for Android, and presented the results of our empirical evaluation of the system on five real apps. The results showed that for our subjects, ACTEve is significantly more efficient than the naive concolic execution technique, referred to as AlLSEQS.

We have at least three important directions for future work. First, ACTEve only alleviates path explosion. The improved efficiency of ACTEvE over ALLSEQS may not be sufficient to handle apps that have significantly more paths than our subjects. An example of such an app is one that has many widgets (e.g., a virtual keyboard). We plan to study other subsumption patterns besides the read-only pattern that we currently exploit to tame path explosion. The independence pattern, described in Appendix $\mathrm{C}$, is an example. 
Second, our system currently handles only one type of events (i.e., tap events). There are many other types of events such as incoming phone calls and gestures. Extending our system to handle other types of events will widen its applicability to more apps. Third, we intend to conduct a more exhaustive empirical evaluation with more subjects to further confirm ACTEve's improvement over AllSEqs.

\section{Acknowledgments}

We thank the anonymous reviewers for insightful comments, and Xin Zhang for helpful discussions. This work was supported in part by a faculty research award from Google, grants from EPSRC and BIS London, and NSF CCF-0541048, NSF CCF-0964647, NSF CCF-1116210, ABB Inc., and an IBM Software Quality Innovation Award.

\section{References}

[1] S. Anand, P. Godefroid, and N. Tillmann. Demanddriven compositional symbolic execution. In TACAS, pages 367-381, 2008.

[2] N. Bjørner, N. Tillmann, and A. Voronkov. Path feasibility analysis for string-manipulating programs. In TACAS, pages 307-321, 2009.

[3] P. Boonstoppel, C. Cadar, and D. R. Engler. RWset: Attacking path explosion in constraint-based test generation. In TACAS, pages 351-366, 2008.

[4] R. C. Bryce, S. Sampath, and A. M. Memon. Developing a single model and test prioritization strategies for event-driven software. TSE, 37(1):48-64, 2011.

[5] C. Cadar, D. Dunbar, and D. R. Engler. KLEE: Unassisted and automatic generation of high-coverage tests for complex systems programs. In OSDI, pages 209$224,2008$.

[6] B.-G. Chun, S. Ihm, P. Maniatis, M. Naik, and A. Patti. Clonecloud: elastic execution between mobile device and cloud. In EuroSys, pages 301-314, 2011.

[7] L. M. de Moura and N. Bjørner. Z3: An efficient SMT solver. In TACAS, pages 337-340, 2008.

[8] W. Enck, P. Gilbert, B. gon Chun, L. P. Cox, J. Jung, P. McDaniel, and A. Sheth. Taintdroid: An information-flow tracking system for realtime privacy monitoring on smartphones. In OSDI, pages 393-407, 2010 .

[9] A. P. Felt, E. Chin, S. Hanna, D. Song, and D. Wagner. Android permissions demystified. In $C C S$, pages $627-$ 638,2011 .

[10] S. R. Ganov, C. Killmar, S. Khurshid, and D. E. Perry. Event listener analysis and symbolic execution for testing GUI applications. In ICFEM, pages 69-87, 2009.

[11] P. Godefroid. Compositional dynamic test generation. In $P O P L$, pages 47-54, 2007.

[12] P. Godefroid. Higher-order test generation. In PLDI, pages 258-269, 2011.
[13] P. Godefroid, A. Kiezun, and M. Y. Levin. Grammarbased whitebox fuzzing. In PLDI, pages 206-215, 2008.

[14] P. Godefroid, N. Klarlund, and K. Sen. DART: Directed automated random testing. In $P L D I$, pages $213-223$, 2005 .

[15] P. Godefroid, M. Y. Levin, and D. A. Molnar. Automated whitebox fuzz testing. In NDSS, 2008.

[16] P. Godefroid and D. Luchaup. Automatic partial loop summarization in dynamic test generation. In ISSTA, pages $23-33,2011$.

[17] S. Khurshid and Y. L. Suen. Generalizing symbolic execution to library classes. In PASTE, pages 103-110, 2005.

[18] R. Majumdar and K. Sen. Hybrid concolic testing. In ICSE, pages 416-426, 2007.

[19] R. Majumdar and R.-G. Xu. Reducing test inputs using information partitions. In $C A V$, pages 555-569, 2009.

[20] A. M. Memon. A comprehensive framework for testing graphical user interfaces. Ph.D., Univ. of Pittsburg, 2001.

[21] A. M. Memon, I. Banerjee, and A. Nagarajan. GUI ripping: Reverse engineering of graphical user interfaces for testing. In Working Conference on Reverse Engineering, pages 260-269, 2003.

[22] A. M. Memon, M. E. Pollack, and M. L. Soffa. Automated test oracles for GUIs. In FSE, pages 30-39, 2000 .

[23] A. M. Memon and M. L. Soffa. Regression testing of GUIs. In ESEC/FSE, pages 118-127, 2003.

[24] D. Octeau, S. Jha, and P. McDaniel. Retargeting android applications to java bytecode. In FSE, 2012.

[25] C. S. Pasareanu, P. C. Mehlitz, D. H. Bushnell, K. Gundy-Burlet, M. R. Lowry, S. Person, and M. Pape. Combining unit-level symbolic execution and systemlevel concrete execution for testing NASA software. In ISSTA, pages 15-26, 2008.

[26] D. Qi, H. D. T. Nguyen, and A. Roychoudhury. Path exploration based on symbolic output. In FSE, pages 278-288, 2011.

[27] R. A. Santelices and M. J. Harrold. Exploiting program dependencies for scalable multiple-path symbolic execution. In ISSTA, pages 195-206, 2010.

[28] P. Saxena, P. Poosankam, S. McCamant, and D. Song. Loop-extended symbolic execution on binary programs. In ISSTA, pages 225-236, 2009 .

[29] K. Sen, D. Marinov, and G. Agha. CUTE: A concolic unit testing engine for C. In ESEC/FSE, pages 263272,2005 .

[30] T. Takala, M. Katara, and J. Harty. Experiences of system-level model-based GUI testing of an Android application. In ICST, pages 377-386, 2011. 
[31] R. Vallée-Rai, L. Hendren, V. Sundaresan, P. Lam, E. Gagnon, and P. Co. Soot - A Java optimization framework. In Conference of the Centre for Advanced Studies on Collaborative Research, pages 125-135, 1999.

[32] M. Veanes, P. de Halleux, and N. Tillmann. Rex: Symbolic regular expression explorer. In ICST, pages 498507,2010

[33] L. J. White and H. Almezen. Generating test cases for GUI responsibilities using complete interaction sequences. In ISSRE, pages 110-123, 2000.

[34] X. Yuan, M. B. Cohen, and A. M. Memon. GUI interaction testing: Incorporating event context. TSE, 37(4):559-574, 2011.

[35] X. Yuan and A. M. Memon. Generating event sequencebased test cases using GUI runtime state feedback. TSE, 36(1), 2010.

\section{APPENDIX}

This appendix consists of the four parts. In Appendix A. we explain the rules for deriving the evaluation relations in our operational semantics. In Appendix B, we prove the lemmas and the theorem presented in the main text of the paper. In Appendix C we present another implementation of pruning that uses the notion of independence. Finally, in Appendix D we present the rest of the graphs for the results in Study 2.

\section{A. RULES FOR EVALUATION RELATIONS}

Rules for the evaluation relations are given in Figure 11 . Most of the rules in the figure follow from our intended reading of all the parts in the evaluation relations. For example, the first rule for the conditional statement says that if the boolean condition $b$ evaluates to true, we extend the $C$ component of a symbolic state with the result $b^{\prime}$ of symbolically evaluating the condition $b$, and follow the true branch.

The only unusual rule is the second one in (3) for evaluating input event sequences. This rule describes how to thread the iterative execution of a program. One unusual aspect is that the symbolic global state and the path constraint are reset for each input event. This ensures that the path constraint of a final concolic state restricts only the current input event, not any previous ones, in the input sequence.

\section{B. PROOFS OF LEMMAS AND THEOREM}

In this part of the appendix, we provide proofs of Lemmas 1 and 2 stated in Section 4.4 and Lemma 3 and Theorem 4 stated in Section 4.5 in the main text of the paper.

\section{B.1 Proof of Lemma 1}

Lemma 1] For sets $T, T^{\prime}$ of feasible traces,

$$
T \sqsubseteq T^{\prime} \Longrightarrow \operatorname{symex}\left(s_{i n}, \gamma_{i n}, T\right) \sqsubseteq \operatorname{symex}\left(s_{i n}, \gamma_{i n}, T^{\prime}\right) \text {. }
$$

Proof. Pick $\tau$ from symex $\left(s_{i n}, \gamma_{i n}, T\right)$. We show show that some $\tau^{\prime}$ in symex $\left(s_{i n}, \gamma_{i n}, T^{\prime}\right)$ satisfies $\tau \sqsubseteq \tau^{\prime}$. By the definition of symex $\left(s_{i n}, \gamma_{i n}, T\right)$, there exist a feasible trace $\alpha$, a path constraint $C$, and a set $W$ of global variables such that

$$
\alpha \in T \wedge \tau=\alpha\langle C, W\rangle
$$

By assumption that $T \sqsubseteq T^{\prime}$, the first conjunct above implies the existence of $\alpha^{\prime} \in T^{\prime}$ satisfying $\alpha \sqsubseteq \alpha^{\prime}$.

We now claim that $\alpha^{\prime}\langle C, W\rangle$ is the desired trace $\tau^{\prime}$. To show this claim, it is sufficient to prove that final $(\alpha\langle C, W\rangle)$ is a subset of final $\left(\alpha^{\prime}\langle C, W\rangle\right)$. The feasibility of $\alpha^{\prime}\langle C, W\rangle$ follows from this. Pick $\gamma_{1}$ from final $(\alpha\langle C, W\rangle)$. Then, there exist $\gamma_{0} \in$ final $(\alpha), n$, and $\Gamma$ such that

$$
\left\langle s_{i n}, n,\left\langle\gamma_{0}, \gamma_{0}, \epsilon\right\rangle\right\rangle \downarrow\left\langle\gamma_{1}, \Gamma, C\right\rangle \triangleright W .
$$

Since final $(\alpha) \subseteq$ final $\left(\alpha^{\prime}\right)$, the global state $\gamma_{0}$ must be in final $\left(\alpha^{\prime}\right)$, meaning that for some $\pi^{\prime}$,

$$
\left\langle s_{i n}, \pi^{\prime}, \gamma_{i n}\right\rangle \Downarrow \gamma_{0} \triangleright \alpha^{\prime} .
$$

From (5) and (4) follows that

$$
\left\langle s_{i n}, \pi^{\prime} n, \gamma_{i n}\right\rangle \Downarrow \gamma_{0} \triangleright \alpha^{\prime}\langle C, W\rangle .
$$

Hence, $\gamma_{1}$ is in final $\left(\alpha^{\prime}\langle C, W\rangle\right)$, as required.

\section{B.2 Proof of Lemma 2}

$$
\begin{aligned}
& \text { LEMma } 2 \text { For all sets } T, T^{\prime} \text { of feasible traces, if } \\
& T \sqsubseteq T^{\prime} \text {, we have that } \\
& \bigcup \begin{array}{l}
\text { branch } \left.\left(\left(\tau_{|\tau|}\right) \cdot C\right) \mid \tau \in \operatorname{symex}\left(s_{i n}, \gamma_{i n}, T\right)\right\} \\
\quad \subseteq \bigcup\left\{\operatorname{branch}\left(\left(\tau_{|\tau|}\right) \cdot C\right) \mid \tau \in \operatorname{symex}\left(s_{i n}, \gamma_{i n}, T^{\prime}\right)\right\} .
\end{array}
\end{aligned}
$$

Proof. We will show that for all $\tau \in \operatorname{symex}\left(s_{i n}, \gamma_{i n}, T\right)$, there exists $\tau^{\prime} \in \operatorname{symex}\left(s_{i n}, \gamma_{i n}, T^{\prime}\right)$ satisfying

$$
\operatorname{branch}\left(\left(\tau_{|\tau|}\right) . C\right) \subseteq \operatorname{branch}\left(\left(\tau_{\left|\tau^{\prime}\right|}^{\prime}\right) \cdot C\right) .
$$

Pick $\tau$ from symex $\left(s_{i n}, \gamma_{i n}, T\right)$. Then, $\tau$ is feasible and has length at least 1 . Also, there exist a feasible trace $\alpha$, a path constraint $C$, and a set of global variables $W$ such that

$$
\tau=\alpha\langle C, W\rangle \wedge \alpha \in T
$$

Since $T \sqsubseteq T^{\prime}$, there should be $\alpha^{\prime} \in T^{\prime}$ with

$$
\alpha \sqsubseteq \alpha^{\prime} .
$$

Let $\tau^{\prime}=\alpha^{\prime}\langle C, R, W\rangle$. It is sufficient to prove that $\tau^{\prime}$ is feasible. Since $\tau$ is feasible and it is $\alpha\langle C, W\rangle$, there exist $n$, $\gamma_{0}, \gamma_{1}$, and $\Gamma_{1}$ such that

$$
\gamma_{0} \in \operatorname{final}(\alpha) \wedge\left\langle s_{i n}, n,\left\langle\gamma_{0}, \gamma_{0}, \epsilon\right\rangle\right\rangle \Downarrow\left\langle\gamma_{1}, \Gamma_{1}, C\right\rangle \triangleright W .
$$

Since $\alpha \sqsubseteq \alpha^{\prime}, \gamma_{0}$ is also in final $\left(\alpha^{\prime}\right)$. This and the second conjunct of (6) imply that $\alpha^{\prime}\langle C, W\rangle$ is feasible.

\section{B.3 Proof of Lemma 3}

Lemma 3 For all sets $\Delta$ of feasible traces, rprune $(\Delta)$ is a subset of $\Delta$ and satisfies the condition in (1).

Proof. Let $\Pi=$ rprune $(\Delta)$. Because of the definition of rprune, $\Pi$ has to be a subset of $\Delta$. It remains to prove that the condition in (1) holds for $\Delta$ and $\Pi$. Pick $\tau$ in $\Delta$. We should find $\tau^{\prime}$ in $\Pi \cup \operatorname{sprefix}(\Delta)$ such that $\tau \sqsubseteq \tau^{\prime \prime}$. If $\tau$ is in $\Pi$, we can choose $\tau$ itself as $\tau^{\prime \prime}$. The condition $\tau \sqsubseteq \tau^{\prime \prime}$ holds because of the reflexivity of $\sqsubseteq$. If $\tau$ is not in $\Pi$, we must have that $|\tau| \geq 1$ and $\left(\tau_{|\tau|}\right) . W=\emptyset$. Let $\alpha$ be the prefix of $\tau$ that has length $|\tau|-1$. Then, $\alpha$ is feasible, and it belongs to sprefix $(\Delta)$. Furthermore, final $(\tau) \subseteq$ final $(\alpha)$, since the additional last step of $\tau$ denotes read-only computations. Hence, $\tau \sqsubseteq \alpha$. From what we have just shown follows that $\alpha$ is the desired feasible trace. 


$$
\begin{aligned}
& \overline{\langle\text { skip, } n, \omega\rangle \downarrow \omega \triangleright \emptyset} \\
& \overline{\langle g=e, n, \omega\rangle \downarrow\left\langle\omega \cdot \gamma\left[g: n^{\prime}\right], \omega \cdot \Gamma\left[g: e^{\prime}\right], \omega \cdot C\right\rangle \triangleright\{g\}} \\
& \left.\frac{\left\langle s_{1}, n, \omega\left[C:(\omega . C) b^{\prime\langle l, t r u e\rangle}\right]\right\rangle \downarrow \omega^{\prime} \triangleright W}{\left\langle\text { if } b^{l} s_{1} \text { else } s_{2}, n, \omega\right\rangle \downarrow \omega^{\prime} \triangleright W} \quad \text { [if } \llbracket b \rrbracket(n, \omega)=\text { true and } \llbracket b \rrbracket^{s}(\omega)=b^{\prime}\right] \\
& \left.\frac{\left\langle s_{2}, n, \omega\left[C:(\omega . C)\left(\neg b^{\prime}\right)^{\langle l, f a l s e\rangle}\right]\right\rangle \downarrow \omega^{\prime} \triangleright W}{\left\langle\text { if } b^{l} s_{1} \text { else } s_{2}, n, \omega\right\rangle \downarrow \omega^{\prime} \triangleright W} \quad \text { [if } \llbracket b \rrbracket(n, \omega)=\text { false and } \llbracket b \rrbracket^{s}(\omega)=b^{\prime}\right] \\
& \frac{\left\langle s_{1}, n, \omega\right\rangle \downarrow \omega^{\prime} \triangleright W \quad\left\langle s_{2}, n, \omega^{\prime}\right\rangle \downarrow \omega^{\prime \prime} \triangleright W^{\prime}}{\left\langle s_{1} ; s_{2}, n, \omega\right\rangle \downarrow \omega^{\prime \prime} \triangleright W \cup W^{\prime}} \\
& \left.\frac{\left\langle s, n, \omega\left[C:(w . C) b^{\prime\langle l, \text { true }\rangle}\right]\right\rangle \downarrow \omega^{\prime} \triangleright W^{\prime} \quad\left\langle\text { while } b^{l} s, n, \omega^{\prime}\right\rangle \downarrow \omega^{\prime \prime} \triangleright W^{\prime \prime}}{\left\langle\text { while } b^{l} s, n, \omega\right\rangle \downarrow \omega^{\prime \prime} \triangleright W^{\prime} \cup W^{\prime \prime}} \quad \text { [if } \llbracket b \rrbracket(n, \omega)=\text { true and } \llbracket b \rrbracket^{s}(\omega)=b^{\prime}\right] \\
& \left.\overline{\left\langle\text { while } b^{l} s, n, \omega\right\rangle \downarrow \omega\left[C:(w \cdot C)\left(\neg b^{\prime}\right)^{\langle l, f a l s e\rangle}\right] \triangleright \emptyset} \quad \text { [if } \llbracket b \rrbracket(n, \omega)=\text { false and } \llbracket b \rrbracket^{s}(\omega)=b^{\prime}\right] \\
& \langle s, \pi, \gamma\rangle \Downarrow \gamma^{\prime} \triangleright \tau \\
& \overline{\langle s, \epsilon, \gamma\rangle \Downarrow \gamma \triangleright \epsilon} \\
& \frac{\langle s, n,\langle\gamma, \gamma, \epsilon\rangle\rangle \downarrow \omega \triangleright W \quad\langle s, \pi, \omega \cdot \gamma\rangle \Downarrow \gamma^{\prime} \triangleright \tau^{\prime}}{\langle s, n \pi, \gamma\rangle \Downarrow \gamma^{\prime} \triangleright\langle\omega \cdot C, W\rangle \tau^{\prime}}
\end{aligned}
$$

Figure 11: Concolic execution semantics.

\section{B.4 Proof of Theorem 4}

TheOREM 4 For every $k \geq 1$,

$\operatorname{branch}\left(\operatorname{ACTEvE}\left(s_{i n}, \gamma_{i n}, k\right)\right)$

$$
=\operatorname{branch}\left(\bigcup_{i=0}^{k} \operatorname{symex}^{i}\left(s_{i n}, \gamma_{i n},\{\epsilon\}\right)\right) \text {. }
$$

Proof. The LHS of the equation is a subset of the RHS, because

$$
\operatorname{ACTEve}\left(s_{i n}, \gamma_{i n}, k\right) \subseteq \bigcup_{i=0}^{k} \operatorname{symex}^{i}\left(s_{i n}, \gamma_{i n},\{\epsilon\}\right)
$$

and the branch operator is monotone with respect to the subset relation. In the remainder of this proof, we show that the RHS is also a subset of the LHS.

Let $F$ be a function on sets of traces given by $F(T)=$ symex $\left(s_{i n}, \gamma_{i n}, T\right)$. Define the operator Ibranch on such trace sets by:

$$
\operatorname{lbranch}(T)=\bigcup\left\{\operatorname{branch}\left(\left(\tau_{|\tau|}\right) \cdot C\right)|\tau \in T \wedge| \tau \mid \geq 1\right\} .
$$

Intuitively, this operator collects every branch covered by the last step of some trace in $T$.

We will use the following three facts that hold for all $j$ in $\{0, \ldots, k-1\}$ :

$$
\begin{gathered}
\bigcup_{i=0}^{j} \Delta_{i} \sqsubseteq \bigcup_{i=0}^{j} \Pi_{i}, \\
\bigcup_{i=0}^{j} F^{i}(\{\epsilon\}) \sqsubseteq \bigcup_{i=0}^{j} \Pi_{i}, \\
\operatorname{branch}\left(\bigcup_{i=0}^{j+1} F^{i}(\{\epsilon\})\right)=\operatorname{lbranch}\left(\bigcup_{i=1}^{j+1} F^{i}(\{\epsilon\})\right) .
\end{gathered}
$$

Here $\Delta_{i}$ and $\Pi_{i}$ are the trace sets that ACTEvE computes. We prove all of these facts simutaneously by induction on $j$.
The base cases are immediate from the definitions of $F^{i}, \Delta_{i}$ and $\Pi_{i}$.

The inductive case of the first fact is proved as follows:

$$
\begin{aligned}
\bigcup_{i=0}^{j+1} \Delta_{i} & =\left(\bigcup_{i=0}^{j} \Delta_{i}\right) \cup \Delta_{j+1} \\
& \sqsubseteq\left(\bigcup_{i=0}^{j} \Delta_{i}\right) \cup \operatorname{sprefix}\left(\Delta_{j+1}\right) \cup \Pi_{j+1} \\
& \subseteq\left(\bigcup_{i=0}^{j} \Delta_{i}\right) \cup \Pi_{j+1} \\
& \sqsubseteq\left(\bigcup_{i=0}^{j} \Pi_{i}\right) \cup \Pi_{j+1}=\left(\bigcup_{i=0}^{j+1} \Pi_{i}\right) .
\end{aligned}
$$

The sprefix $\left(\Delta_{j+1}\right)$ in the first line is the set of all strict prefixes of $\Delta_{j+1}$ (i.e., sprefix $\left(\Delta_{j+1}\right)=\left\{\tau\left|\exists \tau^{\prime}.\right| \tau^{\prime} \mid \geq 1 \wedge \tau \tau^{\prime} \in\right.$ $\left.\left.\Delta_{j+1}\right\}\right)$. The derivation proves $\left(\bigcup_{i=0}^{j+1} \Pi_{i}\right) \sqsubseteq\left(\bigcup_{i=0}^{j+1} \Delta_{i}\right)$, because $T \subseteq T^{\prime}$ implies $T \sqsubseteq T^{\prime}$ and the subsumption $\sqsubseteq$ is reflexive and transitive. Also, the derivation uses only true steps, as it should. The second step holds because prune $\left(\Delta_{j+1}\right)=\Pi_{j+1}$, the result of the prune operation satisfies the subsumption relationship in (1) (Section 4.4), and the union operator is monotone with respect to $\sqsubseteq$. The third step holds because sprefix $\left(\Delta_{j+1}\right) \subseteq \bigcup_{i=0}^{j} \Delta_{i}$. The fourth step follows from the induction hypothesis.

For the inductive case of the second fact, we notice that

$$
\begin{aligned}
& F^{j+1}(\{\epsilon\}) \subseteq F\left(\bigcup_{i=0}^{j} F^{i}(\{\epsilon\})\right) \sqsubseteq F\left(\bigcup_{i=0}^{j} \Pi_{i}\right) \\
& =\bigcup_{i=0}^{j} F\left(\Pi_{i}\right) \quad=\bigcup_{i=1}^{j+1} \Delta_{i} \\
& \subseteq \bigcup_{i=0}^{j+1} \Delta_{i} \quad \sqsubseteq \bigcup_{i=0}^{j+1} \Pi_{i}
\end{aligned}
$$

The first step uses the monotonicity of $F$ with respect to the subset relation, and the second uses the induction hypothesis and the fact that $F$ preserves subsumption (Lemma 1 ). 


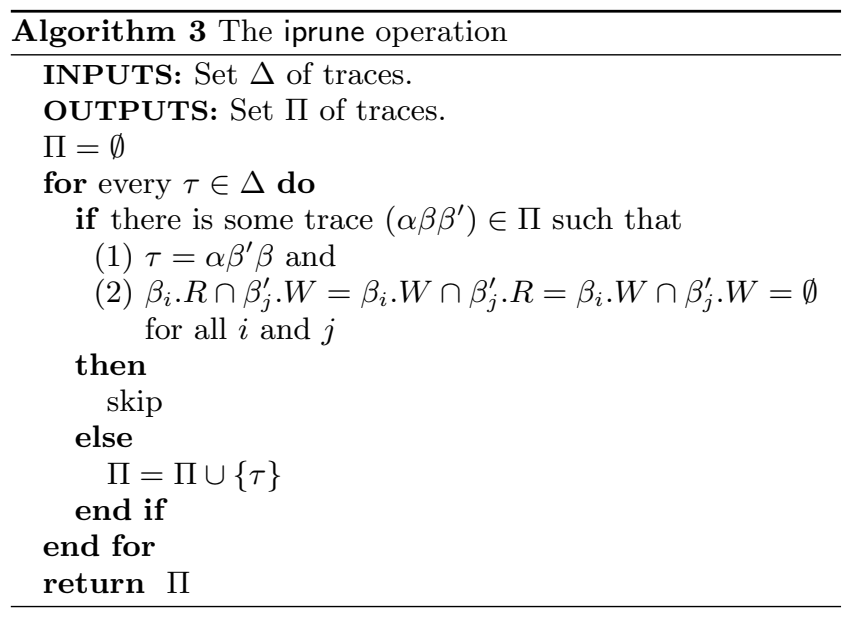

The third holds because $F$ preserves union. The fourth step follows the definition of $\Delta_{i}$, and the last step from the inductive step of the first fact, which we have already proved. Since the relation $\sqsubseteq$ includes the subset relation and is reflexive and transitive, the above derivation shows that $F^{j+1}(\{\epsilon\}) \sqsubseteq \bigcup_{i=0}^{j+1} \Pi_{i}$. Combining this and the induction hypothesis, we get the desired

$$
\bigcup_{i=0}^{j+1} F^{i}(\{\epsilon\})=F^{j+1}(\{\epsilon\}) \cup \bigcup_{i=0}^{j} F^{i}(\{\epsilon\}) \sqsubseteq \bigcup_{i=0}^{j+1} \Pi_{i} .
$$

Here we use the fact that the union operator is monotone with respect to $\sqsubseteq$.

For the inductive case of the third fact, we observe that $\operatorname{branch}\left(F^{j+2}(\{\epsilon\})\right)$ is a subset of

$$
\operatorname{branch}\left(F^{j+1}(\{\epsilon\})\right) \cup \operatorname{lbranch}\left(F^{j+2}(\{\epsilon\})\right) .
$$

This superset itself is included in

$$
\operatorname{lbranch}\left(\bigcup_{i=1}^{j+1} F^{i}(\{\epsilon\})\right) \cup \operatorname{lbranch}\left(F^{j+2}(\{\epsilon\})\right)
$$

because of the induction hypothesis. Using this observation and the induction hypothesis again, we complete the proof of this inductive case as follows:

$$
\begin{aligned}
& \operatorname{branch}\left(\bigcup_{i=0}^{j+2} F^{i}(\{\epsilon\})\right) \\
& =\operatorname{branch}\left(\bigcup_{i=0}^{j+1} F^{i}(\{\epsilon\})\right) \cup \operatorname{branch}\left(F^{j+2}(\{\epsilon\})\right) \\
& \subseteq \operatorname{Ibranch}\left(\bigcup_{i=1}^{j+1} F^{i}(\{\epsilon\})\right) \cup \operatorname{lbranch}\left(F^{j+2}(\{\epsilon\})\right) \\
& =\operatorname{Ibranch}\left(\bigcup_{i=1}^{j+2} F^{i}(\{\epsilon\})\right) .
\end{aligned}
$$

The two equalities here use the fact that Ibranch preserves the union operator.

Using the three facts just shown, we can complete the proof of this theorem as follows:

$$
\begin{aligned}
\operatorname{branch}\left(\bigcup_{i=0}^{k} F^{i}(\{\epsilon\})\right) & =\operatorname{Ibranch}\left(\bigcup_{i=1}^{k} F^{i}(\{\epsilon\})\right) \\
& =\operatorname{lbranch} \circ F)\left(\bigcup_{i=0}^{k-1} F^{i}(\{\epsilon\})\right) \\
& \subseteq \operatorname{Ibranch} \circ F)\left(\bigcup_{i=0}^{k-1} \Pi_{i}\right) \\
& =\operatorname{Ibranch}\left(\bigcup_{i=0}^{k-1} F\left(\Pi_{i}\right)\right) \\
& =\operatorname{Ibranch}\left(\bigcup_{i=1}^{k} \Delta_{i}\right) \\
& \subseteq \operatorname{Ibranch}\left(\operatorname{ACTEVE}\left(s_{i n}, \gamma_{i n}, k\right)\right) .
\end{aligned}
$$

The first step is by the third fact, and the second and fourth steps hold because $F$ preserves the union operator. The third step follows from the second fact and Lemma 2 The last two steps are just the unrolling of the definitions of $\Delta_{i}$ and the result of $\operatorname{ACTEvE}\left(s_{i n}, \gamma_{i n}, k\right)$.

\section{INDEPENDENCE-BASED PRUNING}

Algorithm 3 gives another implementation of prunning, called iprune, which exploits a form of independence. This implementation assumes that the evaluation relations track a set of read global variables, in addition to written ones. This means that the forms of evaluation relations are changed to

$$
\langle s, n, \omega\rangle \downarrow \omega^{\prime} \triangleright W, R \quad\langle s, \pi, \gamma\rangle \Downarrow \gamma^{\prime} \triangleright \tau,
$$

where $R$ is a set of read variables and $\tau$ is now a sequence of triples $C, W, R$. Also, the rules for these relations are changed appropriately. Lemmas 1 and 2 in Section 4.4 and Theorem 4 in Section 4.6 remain valid even with these changes.

The iprune operator detects two traces $\tau, \tau^{\prime}$ in $\Delta$ such that $\tau$ can be obtained by swapping independent consecutive parts in $\tau^{\prime}$. In Figure 3, $\alpha \beta \beta^{\prime}$ corresponds to $\tau^{\prime}$, and $\beta$ and $\beta^{\prime}$ represent consecutive independent parts. Although the iprune operator is not implemented in our system, it illustrates the generality of using our subsumption condition. The following lemma shows that iprune satisfies the condition.

Lemma 5. The result of iprune is a subset of its input trace set, and it always satisfis the subsumption relationship in (1) (Section 4.5).

Proof. Consider a set $\Delta$ of feasible traces, and let $\Pi=$ iprune $(\Delta)$. From the definition of iprune, it is immediate that $\Pi$ is a subset of $\Delta$. To prove that $\Pi$ also satisfies the condition in (1), pick $\tau$ from $\Delta$. We will have to find $\tau^{\prime}$ in $\Pi$ such that $\tau \sqsubseteq \tau^{\prime}$. If $\tau$ is aleady in $\Pi$, we can just use $\tau$ for $\tau^{\prime}$. Suppoes that $\tau$ is not in $\Pi$. Then, by the definition of our algorithm, there must be a feasible trace $\left(\alpha \beta \beta^{\prime}\right)$ in $\Pi$ such that (1) $\tau=\alpha \beta^{\prime} \beta$ and (2) for all $i$ and $j$,

$\left(\beta_{i}\right) \cdot R \cap\left(\beta_{j}^{\prime}\right) \cdot W=\left(\beta_{i}\right) \cdot W \cap\left(\beta_{j}^{\prime}\right) \cdot R=\left(\beta_{i}\right) \cdot W \cap\left(\beta_{j}^{\prime}\right) \cdot W=\emptyset$.

Since $\Pi$ is a subset of $\Delta$ throughout the execution of iprune, we know that $\alpha \beta \beta^{\prime}$ is a feasible trace. Furthermore, the two properties of this trace above imply that

$$
\operatorname{final}(\tau)=\operatorname{final}\left(\alpha \beta \beta^{\prime}\right),
$$

so $\tau \sqsubseteq\left(\alpha \beta \beta^{\prime}\right)$. From what we have just proven so far follows that $\alpha \beta \beta^{\prime}$ is the trace $\tau^{\prime}$ that we are looking for.

\section{RESULTS OF STUDY 2}

In Study 2 (Section 5.4), we presented the results for one of the subject apps. In this part of the Appendix, we present the results for the rest of the subject apps.

The graphs in Figure 12 provide these results. As the graphs show, the results are similar to those discussed in Section 5.4 and show the same reduction in feasible paths explored and constraints checked of ACTEve over AlLSEQS. 

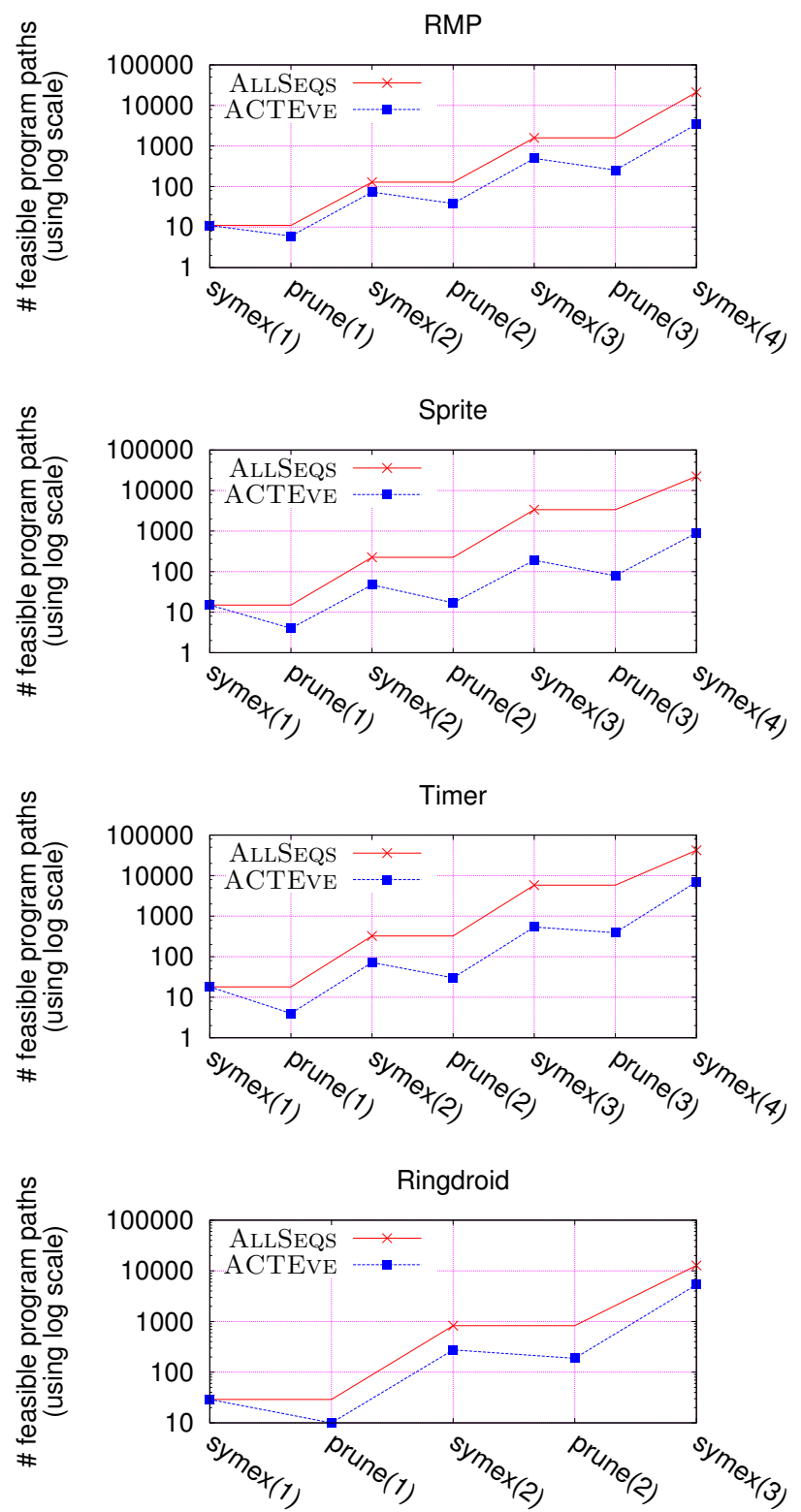

Figure 12: Results of Study 2: The number of paths (using a logarithmic scale) after symex and prune operations in each iteration. Because AllSEqS does not terminate for Timer and Ringdroid when $k=4$, the reported final numbers of paths for those two apps correspond to the time when the time-limit (12-hours) was met. 